\title{
Meta-analysis of prednisone in withdrawal therapy following medication overuse headache
}

\author{
Wenmiao Jie, Jianzhong Xiong, Xiaohua Yan \\ Department of Neurology, People's Hospital in Pingxiang, Pingxiang, China
}

\begin{abstract}
The objective of this meta-analysis was to evaluate the therapeutic effectiveness of prednisone in withdrawal therapy following medication overuse headache. The Cochrane, PubMed, EMBASE, Web of Science, CNKI, VIP, and Wanfang data were searched to identify randomized control trials of prednisone for the treatment of medication overuse headache. Two researchers independently screened published studies according to inclusion and exclusion criteria, and evaluated the methodological quality of included studies. Revman 5.3 software was used to analyze the extracted data, and a total of six randomized control trials involving 510 patients were included in the study. Meta-analysis indicated that there was no significant difference in the ratio of taking painkillers again after withdrawal ( $\mathrm{RR}=0.89,95 \% \mathrm{CI}: 0.70,1.14, P=0.36)$ compared with the control group. There was also no significant difference between the experimental group and the control group in the incidence of withdrawal reactions ( $\mathrm{RR}=1.28,95 \% \mathrm{CI}: 0.87,1.89, P=0.21)$, severity of headache $(\mathrm{RR}=1.56,95 \%$ CI: $-4.83,7.95, P=0.63)$ and the frequency of headache attacks after withdrawal $(\mathrm{RR}=0.14,95 \% \mathrm{CI}$ : $-0.35,0.63, P=0.58$ ). It is concluded that prednisone does not alleviate symptoms in patients with medication overuse headache after withdrawal.
\end{abstract}

Keywords: Prednisone; medication overuse headache; withdrawal therapy; meta-analysis

\section{INTRODUCTION}

Medication overuse headache $(\mathrm{MOH})$ is a chronic headache caused by the frequent use of painkillers by patients. $\mathrm{MOH}$ is a secondary headache which often develops from migraine, tension headache and other types of headaches. The clinical manifestations of medication overuse headache are diverse. The location of the headache, the nature of the headache, the severity of the headache and the associated symptoms vary from patient to patient. Even if the same patient has different episodes, the headaches can vary greatly. This variation often leads to clinical misdiagnosis, which makes treatment of MOH difficult. With recurring headaches, a high disability rate and a high economic burden, $\mathrm{MOH}$ involves substantial adverse impact on patient and their families. The withdrawal from painkillers is a major factor in the success of the treatment for patients with $\mathrm{MOH} .{ }^{1}$ Studies indicate that the use of prednisone can reduce various symptoms of discomfort after withdrawal painkillers in those $\mathrm{MOH}$ patients. Currently, there are a number of related studies on the use of prednisone for withdrawal in China and abroad. However, due to the small sample sizes, different methodologies and varying research quality, the effectiveness of prednisone in treating $\mathrm{MOH}$ remains unclear. To further clarify the effectiveness of prednisone in alleviating the symptoms of patients with $\mathrm{MOH}$ after withdrawal we conducted a meta-analysis on existing randomized controlled trials (RCTs) to evaluate evidence for the clinical application of prednisone in the treatment of $\mathrm{MOH}$.

\section{METHODS}

\section{Database and search strategy}

The PubMed, Cochrane Library, EMBASE, Web of Science, Wanfang, CNKI and VIP databases were searched for RCTs evaluating the effectiveness of prednisone in the treatment of $\mathrm{MOH}$. Manual retrieval was used as required. The main search keywords were "prednisone", and "medication overuse headache". The search terms were either "prednisone" or "prednisolone" and "medication overuse headaches". The citation list of the retrieved literature was manually filtered to 
ensure the validity of the search strategy. The date of the most recent search was December 30, 2020. A detailed retrieval strategy is given in Figure 1.

\section{Inclusion Criteria}

All RCTs of prednisone in treating $\mathrm{MOH}$ were included. The diagnostic criteria of $\mathrm{MOH}$ were: A. Headache present on $\geq 15$ days per month. B. Regular overuse for more than 3 months of one or more drugs that can be taken for acute and/or symptomatic treatment of headache. C. Headache has developed or markedly worsened during medication-overuse. Study subjects were patients diagnosed with $\mathrm{MOH}$, regardless of race or gender. The experimental group was administered prednisone treatment and the control groups were given either celecoxib or placebo or no treatment. The main outcomes were the ratio of taking painkillers again after withdrawal, the frequency of headache, the severity of headache, the incidence of withdrawal reactions.

\section{Exclusion criteria}

The exclusion criteria included: 1 . Original data missing. 2. Full text unobtainable, 3. Treatment plan unclear. 4. Duplication of previously published data.

\section{Literature screening and data extraction}

Two researchers independently selected literature from the titles and abstracts of the primary articles. Publications that did not meet the requirements were excluded from full text reading. Information was extracted independently by two authors using predefined data abstraction forms. The following details were extracted: publication data (authors, publication year, sample size), treatment protocol, treatment duration and main outcomes. After information was extracted, cross-checking was performed. Any disagreement was resolved by a third party. Cohen's kappa was used to assess inter-reviewer agreement.

\section{Evaluating the quality of studies}

The quality of the studies included was evaluated through an assessment tool recommended by Cochrane. Literature quality was evaluated by: random sequence generation, blinding of participants and outcome assessment, allocation concealment, incomplete outcome data, selective reporting and other bias. Each item had three grades of bias risk: low, high and unclear. Differences of opinion between the two reviewers were settled through discussion. If required, a third author arbitrated.

\section{Statistical Methods}

Discontinuous and continuous data were respectively summarized by relative risk (RR) and mean difference (MD). Revman 5.3

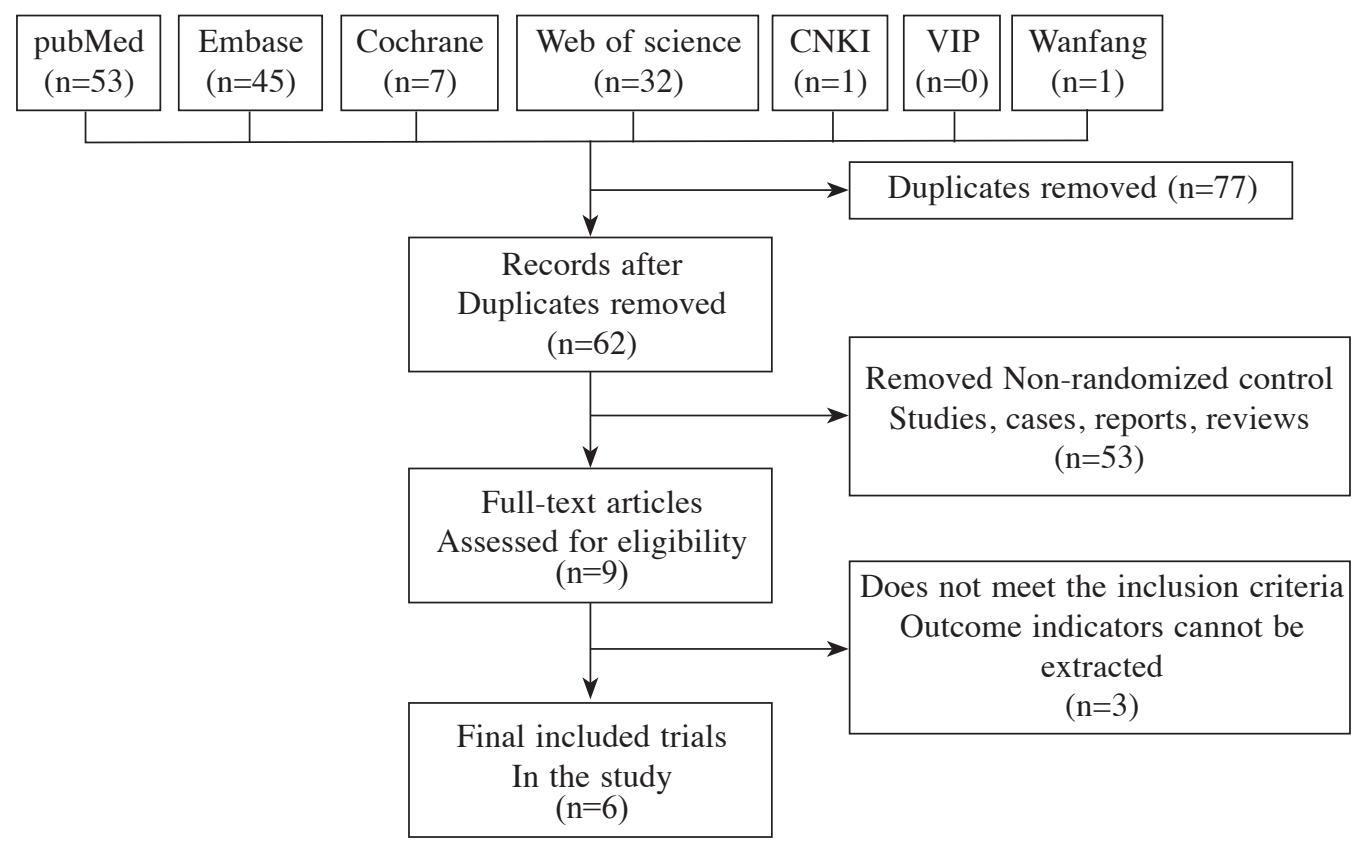

Figure 1. Flowchart gives progress of study selection 
Table 1: Basic features of included trials

\begin{tabular}{|c|c|c|c|c|c|c|}
\hline \multirow{2}{*}{ Included studies } & \multirow{2}{*}{ Nature } & \multicolumn{2}{|c|}{ Sample size } & \multicolumn{2}{|c|}{ Intervention measures } & \multirow{2}{*}{$\begin{array}{l}\text { Outcome } \\
\text { indicators }\end{array}$} \\
\hline & & Experimental & Control & Experimental & Control & \\
\hline Rossil $2006^{3}$ & Italy & 34 & 37 & prednisone & Blank & (3) \\
\hline Boe $2007^{4}$ & Norway & 49 & 48 & prednisone & Placebo & (1) \\
\hline Rabe $2013^{5}$ & Germany & 37 & 44 & prednisone & Placebo & (2) \\
\hline Mansoueh2014 & Iran & 50 & 53 & prednisone & Celecoxib & (1) (4) \\
\hline Taghdiri $2015^{7}$ & Iran & 38 & 42 & prednisone & Celecoxib & (1) (3) (4) \\
\hline Xiong $2015^{8}$ & China & 37 & 41 & prednisone & Placebo & (1) (2) (3) \\
\hline
\end{tabular}

Outcome indicators were: (1) Ratio of taking painkillers again after withdrawal (2) Incidence of withdrawal reactions (3) Severity of headache after withdrawal (4) Frequency of headache after withdrawal

software was employed for data analysis. Data was assessed by either a fixed or a random effect model and statistical heterogeneity was examined by testing $I^{2}$. The fixed effect model was used where heterogeneity was low $\left(I^{2}<50 \%\right)$, while the random effect model was used where heterogeneity was high $\left(I^{2} \geq 50 \%\right)$.

\section{RESULTS}

\section{Description of included studies}

A preliminary search of the literature databases identified 139 publications. Following duplicate removal, only 62 remained, of which a further 53 were removed subsequent to reading their titles and abstracts. The remaining 9 publications were verified according to the inclusion criteria. Following discussion among researchers, 6 of these 9 articles were included. Cohen's kappa was used to assess inter-reviewer agreement and a kappa coefficient of 0.737 was obtained. Details of the research flow path are given in Figure 1.

In summary, this study analyzed six studies involving 510 patients, of whom 245 patients were distributed to the experimental group and 265 to the control group. The samples sizes in those trials ranged from 71 to 103 . The literature included was published between 2006 and 2015. Of the six studies ${ }^{3-8}$ included, two trials ${ }^{6,7}$ reported the frequency of headache attacks, two trials $\mathrm{s}^{3,7}$ reported the severity of headache, two trials ${ }^{5,8}$ reported the incidence of withdrawal reactions, four trials ${ }^{4,6-8} \mathrm{r}$ reported the ratio of taking painkillers again after withdrawal. Features of the six studies included are given in Table 1.

All trials reported on participants randomization. Patients in six studies were divided into the experimental group or control group using computer randomization. Only one of the 6 trials failed to use allocation concealment.One trial did not used blind method; the other five trials used a double-blind method. Five trials mentioned that blind method was used in outcome assessment. One trial did not use blind method in the outcome assessment. All trials were assessed as being at low risk of bias in incomplete outcome data. Selective reporting bias and other bias were evaluated as low risk in all trials. The risk of bias of the six included studies is given in Figure 2.

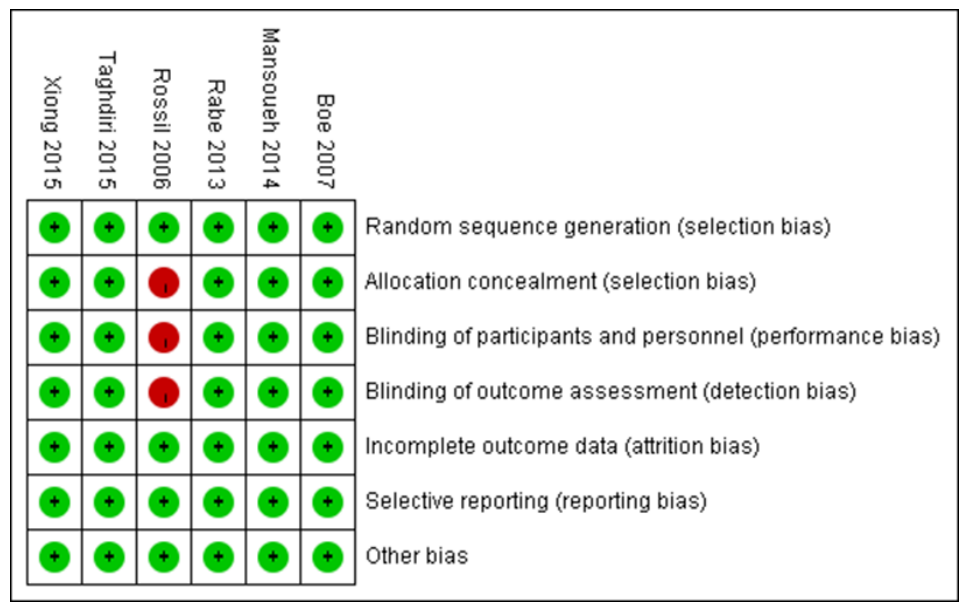

Figure 2. Summary of the risk of bias assessment of including studies. 


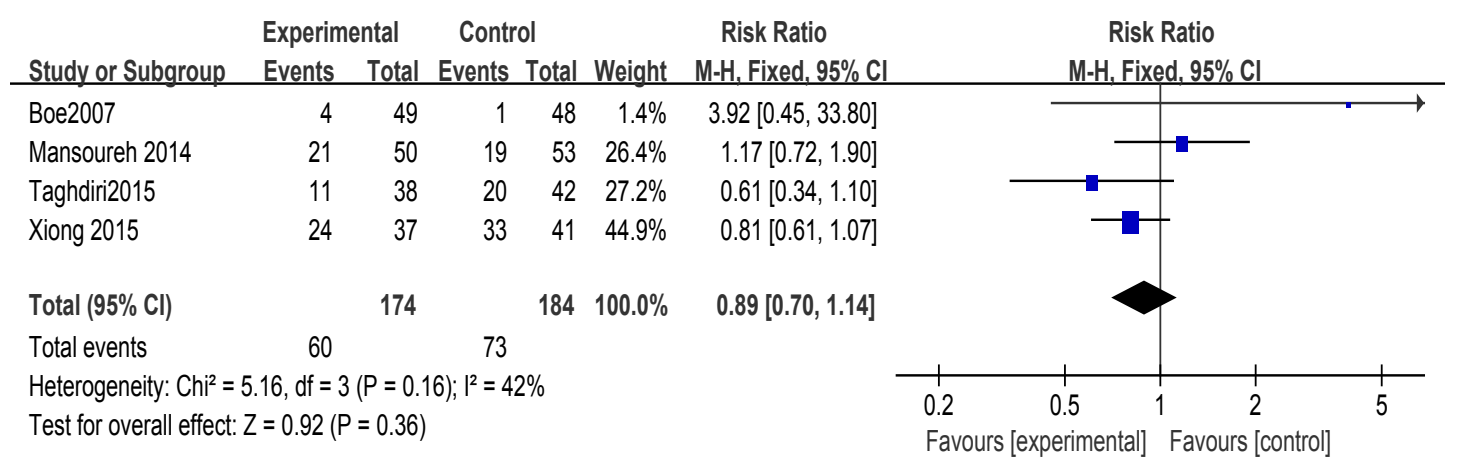

Figure 3. Ratio of taking painkillers again after withdrawal of experimental and control groups by forest plot.

The ratio of taking painkillers again after withdrawal

Four trials ${ }^{4,6-8}$ that reported the ratio of taking painkillers again after withdrawal included 174 participants in the experimental group and 184 participants in the control group. A fixed effect model was used due to insignificant heterogeneity in the data $\left(\mathrm{I}^{2}=42 \%, p=0.16\right)$. Pooled analysis revealed no significant difference in the ratio of taking painkillers again after withdrawal between the experimental group and the control group (RR $=0.89,95 \% \mathrm{CI}: 0.70,1.14, p=0.36)$. These data are presented in Figure 3.

\section{The incidence of withdrawal reactions}

Two trials ${ }^{5,8}$ that reported the incidence of withdrawal reactions included 72 participants in the experimental group and 82 participants in the control group. A fixed effect model was used because the heterogeneity of the data was insignificant $\left(I^{2}=5 \%, p=0.31\right)$. Pooled analysis indicated no significant difference in the incidence of withdrawal reactions between the experimental group and the control group $(\mathrm{RR}=0.89,95 \% \mathrm{CI}$ : $0.70,1.75, p=0.66)$. These data are presented in Figure 4.

\section{Severity of headache after withdrawal}

Two trials ${ }^{[3,7]}$ that reported the severity of headache after withdrawal included 81 participants in the experimental group and 78 participants in the control group. A fixed effect model was used due to lack of heterogeneity in the data $\left(I^{2}=3 \%, p\right.$ $=0.31$ ). Pooled analysis indicated no significant difference in the severity of headache after withdrawal between the experimental group and the control group $(\mathrm{RR}=1.56,95 \% \mathrm{CI}:-4.83,7.59$, $p=0.63)$. These data are presented in Figure 5 .

\section{Frequency of headache attacks after withdrawal}

Two trials ${ }^{6,7}$ that reported the frequency of headache attacks after withdrawal included 88 participants in the experimental group and 95 participants in the control group. A fixed effect model was used due to insignificant heterogeneity in the data $\left(I^{2}=0 \%, p=0.97\right)$. Pooled analysis revealed no significant difference in the frequency of headache attacks after withdrawal between the experimental group and the control group $(\mathrm{RR}=$ $0.14,95 \% \mathrm{CI}:-0.35,0.63, p=0.58)$. These data are presented in Figure 6.

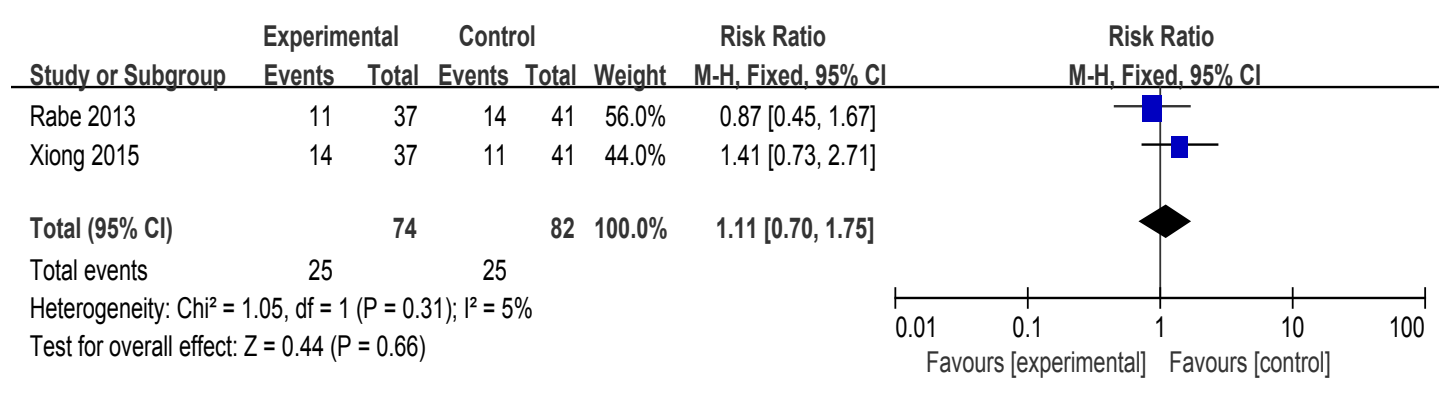

Figure 4. The incidence of withdrawal reactions of experimental and control groups by forest plot. 


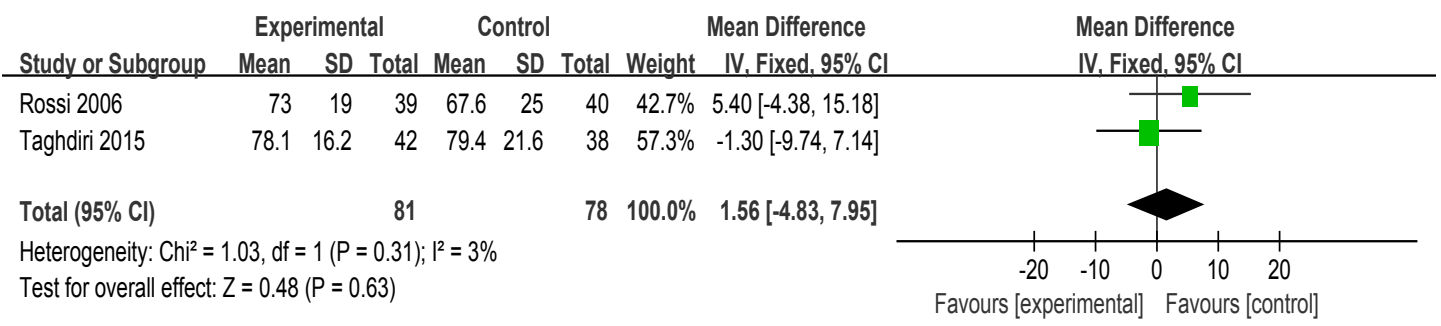

Figure 5. Severity of headache after withdrawal of experimental and control groups by forest plot.

\section{DISCUSSION}

$\mathrm{MOH}$ is caused by the overuse of painkillers. Although $\mathrm{MOH}$ may not be life-threatening, it is a chronic disabling disease. The effect of $\mathrm{MOH}$ on the quality of daily living is far greater than that of migraine. ${ }^{9} \mathrm{MOH}$ is the third most common type of headache after tension headache and migraine, with a prevalence of $0.5 \%$ to $2.6 \% .^{10} \mathrm{MOH}$ is presently hypothesized to be related to the following: (1) genetic factors. ${ }^{11}(2)$ neurotransmitter and endocrine dysfunction. ${ }^{12-16}$ (3) increased levels of neuronal excitability in patients..$^{17}$ (4) abnormal brain function and structure..$^{18-19}(5)$ increased release of calcitonin gene peptide. ${ }^{20-21}$

Health education, withdrawal therapy and use of prophylactic drugs are commonly used in the treatment of $\mathrm{MOH} .{ }^{22-23}$ Due to a lack of health knowledge, patients frequently use excessive amounts of painkillers. ${ }^{24}$ Discontinuation of painkillers is a key measure in treating $\mathrm{MOH}^{25}$ Studies have shown that the frequency and severity of headache attacks can be significantly reduced after cessation of painkillers. Patients with $\mathrm{MOH}$ may experience withdrawal symptoms such as nausea, vomiting, anxiety, sleep disorders, headache, hypotension, tachycardia and other symptoms after stopping analgesics. Treatment of withdrawal can reduce symptoms following discontinuation of painkillers. Recent studies have shown that the use of prednisone for withdrawal can dramatically reduce symptoms in postwithdrawal $\mathrm{MOH}$ patients. ${ }^{26}$
Six randomized controlled studies of prednisone for $\mathrm{MOH}$ related withdrawal were included in this meta-analysis. Four studies analyzed the effectiveness of prednisone on the ratio of taking painkillers again after withdrawal. The pooled results indicated that there was no significant difference in the ratio of taking painkillers again after withdrawal between the experimental and control groups. Two studies analyzed the effectiveness of prednisone therapy on the incidence of withdrawal reactions in patients with $\mathrm{MOH}$. The pooled results revealed no statistically significant difference in the incidence of withdrawal reactions between the experimental and control groups. Two studies analyzed the effectiveness of prednisone withdrawal therapy on the frequency of headache attacks and two studies analyzed the severity of headache following withdrawal in $\mathrm{MOH}$ patients. Our meta-analysis indicates no significant difference in the frequency and severity of headache in patients with $\mathrm{MOH}$ between the experimental and control groups.

In conclusion, prednisone withdrawal therapy has no statistically significant impact in the ratio of taking painkillers again, the incidence of withdrawal reactions or the frequency and severity of headache in $\mathrm{MOH}$ patients after withdrawal compared with the control group. The six studies in this study were completed in five different countries and five of them were high-quality RCTs. Therefore, our study provides valid conclusions to guide the treatment of $\mathrm{MOH}$.

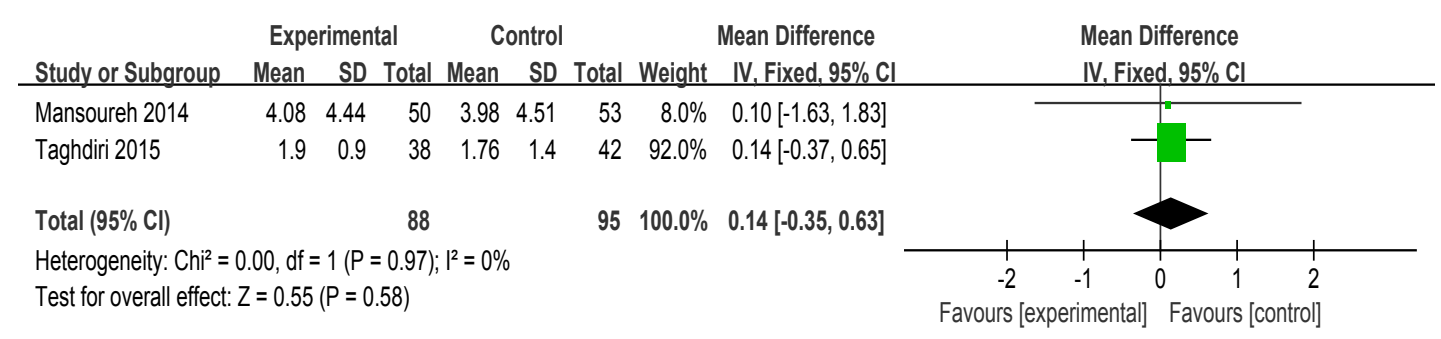

Figure 6. Frequency of headache attacks after withdrawal of experimental and control groups by forest plot. 


\section{REFERENCES}

1. Giamberardino MA, Mitsikostas DD, Martelletti P. Update on medication-overuse headache and its treatment. Curr Treat Options Neurol 2015; 17(8):368.

2. Schmid CW, Maurer K, Schmid DM, et al. Prevalence of medication overuse headache in an interdisciplinary pain clinic. J Headache Pain 2013; $14: 4$

3. Rossi P, Di LC, Faroni J, Cesarino F, Nappi G. Advice alone vs. structured detoxification programmes for medication overuse headache: a prospective, randomized, open-label trial in transformed migraine patients with low medical needs. Cephalalgia 2006; 26(9):1097-105.

4. Bøe MG, Mygland A, Salvesen R. Prednisolone does not reduce withdrawal headache: a randomized, double-blind study. Neurology 2007; 69(1):26-31.

5. Rabe K, Pageler L, Gaul C, et al. Prednisone for the treatment of withdrawal headache in patients with medication overuse headache: a randomized, double-blind, placebo-controlled study. Cephalalgia 2013; 33(3):202-7.

6. Mansoureh T, Mohammad R, et al. Celecoxib versus prednisolone in medication overuse headache withdrawal treatment: double blind randomized clinical trial. Tehran University Med J 2014;71(12):780-6.

7. Taghdiri F, Togha M, Razeghi JS, Paknejad SM. Celecoxib vs prednisone for the treatment of withdrawal headache in patients with medication overuse headache: a randomized, double-blind clinical trial. Headache 2015; 55(1):128-35.

8. Xiong KJ, Wang H. Randomized double-blind controlled study of prednisone in treatment of withdrawal headache in patients with medication overuse headache. Chinese J Biochem Pharmaceut 2015; 3:122-4. (In Chinese)

9. Headache Classification Committee of the International Headache Society (IHS) The International Classification of Headache Disorders, 3rd edition. Cephalalgia 2018; 38(1): 1-211.

10. Westergaard ML, Hansen EH, Glümer C, Olesen J, Jensen RH. Definitions of medication-overuse headache in population-based studies and their implications on prevalence estimates: a systematic review. Cephalalgia 2014; 34(6):409-25.

11. Cevoli S, Sancisi E, Grimaldi D, et al. Family history for chronic headache and drug overuse as a risk factor for headache chronification. Headache 2009; 49(3):412-8.

12. Ayzenberg I, Obermann M, Oberman M, et al. Increased activity of serotonin uptake in platelets in medication overuse headache following regular intake of painkillers and triptans. $J$ Headache Pain 2008; 9(2):109-12.

13. Srikiatkhachorn A. Pathophysiology of chronic daily headache. Curr Pain Headache Rep 2001; 5(6):53744.

14. Rossi C, Pini LA, Cupini ML, Calabresi P, Sarchielli P. Endocannabinoids in platelets of chronic migraine patients and medication-overuse headache patients: relation with serotonin levels. Eur J Clin Pharmacol 2008; 64(1):1-8.

15. Akerman S, Holland PR, Goadsby PJ. Cannabinoid (CB1) receptor activation inhibits trigeminovascular neurons. J Pharmacol Exp Ther 2007.320(1):64-71.

16. Sarchielli P, Rainero I, Coppola F, et al. Involvement of corticotrophin-releasing factor and orexin-A in chronic migraine and medication-overuse headache: findings from cerebrospinal fluid. Cephalalgia 2008; 28(7):714-22.

17. Zappaterra M, Guerzoni S, Cainazzo MM, Ferrari A, Pini LA. Basal cutaneous pain threshold in headache patients. J Headache Pain 2011; 12(3):303-10.

18. Riederer F, Gantenbein AR, Marti M, Luechinger R, Kollias S, Sándor PS. Decrease of gray matter volume in the midbrain is associated with treatment response in medication-overuse headache: possible influence of orbitofrontal cortex. J Neurosci 2013; 33(39):15343-9.

19. Riederer F, Marti M, Luechinger R, et al. Grey matter changes associated with medication-overuse headache: correlations with disease related disability and anxiety. World J Biol Psychiatry 2012; 13(7): 517-25.

20. Ayzenberg I, Obermann M, Nyhuis P, et al. Central sensitization of the trigeminal and somatic nociceptive systems in medication overuse headache mainly involves cerebral supraspinal structures. Cephalalgia 2006; 26(9): 1106-14.

21. Johnson JL, Hutchinson MR, Williams DB, Rolan P. Medication-overuse headache and opioidinduced hyperalgesia: A review of mechanisms, a neuroimmune hypothesis and a novel approach to treatment. Cephalalgia 2013; 33(1):52-64.

22. Estemalik E, Tepper S. Preventive treatment in migraine and the new US guidelines. Neuropsychiatr Dis Treat 2013; 9:709-20.

23. Saper JR, Da SAN. Medication overuse headache: history, features, prevention and management strategies. CNS Drugs 2013; 27(11):867-77.

24. Tepper D. Medication overuse headache. Headache 2017; 57(5):845-6.

25. Diener HC, Antonaci F, Braschinsky M, et al. European Academy of Neurology guideline on the management of medication-overuse headache. Eur J Neurol 2020; 27(7):1102-16.

26. L Pageler, Z Katsarava, HC Diener, et al. Prednisone vs placebo in withdrawal therapy following medication overuse headache. Cephalagia 2007;28:152-6. 